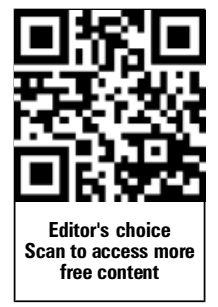

- Additional supplementary files are published online only. To view these files please visit the journal online (http://dx.doi. org/10.1136/annrheumdis2012-202571).

1 Department of Clinical Sciences Lund, Section of Rheumatology, Lund University and Skåne University Hospital, Lund, Sweden

${ }^{2}$ Department of Orthopedics, Lund University, Lund, Sweden ${ }^{3}$ School of Business and Engineering, Department of Exercise Physiology, Biomechanics and Health, Halmstad University, Halmstad, Sweden

${ }^{4}$ Clinical Epidemiology Research \& Training Unit, Boston University School of Medicine, Boston,

Massachusetts, USA

\section{Correspondence to}

Sofia Hagel, Department of Clinical Sciences Lund,

Section of Rheumatology, Lund University and Skåne University Hospital, SE-221 85 Lund, Sweden; sofia.hagel@med.lu.se

Received 27 August 2012 Revised 12 October 2012 Accepted 18 November 2012 Published Online First 8 December 2012

\section{SLinked}

- http://dx.doi.org/10.1136/ annrheumdis-2012-202787

\title{
Trends in the first decade of 21st century healthcare utilisation in a rheumatoid arthritis cohort compared with the general population
}

\author{
Sofia Hagel, ${ }^{1}$ Ingemar F Petersson, ${ }^{2}$ Ann Bremander, ${ }^{1,3}$ Elisabet Lindqvist, ${ }^{1}$ \\ Charlotte Bergknut, ${ }^{2}$ Martin Englund ${ }^{2,4}$
}

\section{ABSTRACT}

Purpose To study 21st century trends in healthcare utilisation by patients with rheumatoid arthritis (RA) compared with the general population.

Methods Observational cohort study. Using Swedish healthcare register data, we identified 3977 Region Skåne residents (mean age in 2001, 62.7 years; 73\% women) presenting with RA (International Classification of Diseases-10 codes M05 or M06) in 1998-2001. We randomly sampled two referents from the general population per RA patient matched for age, sex and area of residence. We calculated the year 2001-2010 trends for the annual ratio (RA cohort/referents) of the mean number of hospitalisations and outpatient clinic visits. Results By the end of the 10 -year period, $62 \%$ of patients and $74 \%$ of referents were still alive and resident in the region. From 2001 to 2010, the ratio (RA cohort/ referents) of the mean number of hospitalisations for men and women decreased by $27 \%(p=0.01)$ and $28 \%$ $(p=0.004)$, respectively. The corresponding decrease was $29 \%(p=0.005)$ and $16 \%(p=0.004)$ for outpatient physician care, $34 \%(p=0.009)$ and $18 \%(p=0.01)$ for nurse visits, and $34 \%(p=0.01)$ and $28 \%(p=0.004)$ for physiotherapy. The absolute reduction in number of hospitalisations was from an annual mean of 0.79 to 0.69 in male patients and from 0.71 to 0.59 in female patients. The corresponding annual mean number of consultations in outpatient physician care by male and female RA patients changed from 9.2 to 7.7 and from 9.9 to 8.7 , respectively.

Conclusions During the first decade of the 21st century, coinciding with increasing use of earlier and more active RA treatment including biological treatment, overall inpatient and outpatient healthcare utilisation by a cohort of patients with RA decreased relative to the general population.

\section{INTRODUCTION}

Since late 20th century, there have been a number of new effective pharmacological treatment options for patients with rheumatoid arthritis (RA). This has led to better control of inflammation, less structural joint damage, and reduced symptoms. Thus, earlier diagnosis, earlier and more active pharmacological treatment, ${ }^{1}$ 'treat to target' strategies, ${ }^{2}$ and the implementation of new treatment guidelines, ${ }^{3}$ as well as the use of biological agents early in the disease course, has changed the treatment paradigm for RA over the first decade of the 21 st century. ${ }^{1}$ This may potentially have changed the need for healthcare in patients with RA unrelated to other major changes in the healthcare system affecting all patients.

In Sweden, $77-92 \%$ of patients receiving diseasemodifying antirheumatic drugs use methotrexate. ${ }^{4}$ On a national level, $\sim 20 \%$ are receiving biological agents. ${ }^{5}$ Patients with early RA treated according to new regimens could be expected to have less impairment of function and work ability ${ }^{6}$ and fewer hospitalisations. ${ }^{8} 9$ However, screening and close monitoring of patients is also necessary when applying the new treatment regimens, ${ }^{10}$ which may impact on healthcare utilisation. Although there are several added benefits from the new treatments, ${ }^{11-13}$ it is difficult to predict what the future will bring in the long term in RA-related healthcare, since evidence on actual healthcare utilisation is scarce and to some extent conflicting. ${ }^{14}$

Our objective, using comprehensive observational register data, was to analyse healthcare utilisation with a focus on rheumatology and orthopaedics over the last decade in a closed cohort of patients with RA compared with a reference cohort from the general population.

\section{METHODS}

The Skåne Healthcare Register

In Sweden, healthcare is based on a tax-financed system and is free of charge (except for a minor co-payment). All healthcare providers, both public and private, are required to submit information for reimbursement purposes. In the Region Skane, the southernmost county of Sweden, with one-eighth of the Swedish population, all inpatient and outpatient visits are registered in the Skåne Healthcare Register (SCHR) by the patient's personal identifier. ${ }^{15}$ For all healthcare providers, date of visit and information on healthcare provider is recorded. For public care, diagnostic codes are registered according to the International Classification of Diseases (ICD) 10 system.

The national population register

Vital events (date of birth and death, marriage and change of residential address) of all inhabitants of Sweden are registered in the population register by the personal identification number. 


\section{Cohort definitions}

\section{RA cohort}

Using SCHR data for the period 1998 to 2001, we identified all adult ( $\geq 18$ years) female or male residents of the Region Skåne who had received a diagnosis of seropositive or other RA (ICD-10 codes M05 and/or M06) on at least two separate occasions, at least one of which was by a specialist in rheumatology or internal medicine (or under specialty training in those disciplines).

\section{Reference cohort}

Using the population register at the end of the year 2000, we randomly sampled two referents from the general population per RA patient matched by birth year, sex and area of residence.
Survival and healthcare utilisation

Using the population register, we traced residence status and survival for each subject (in both the RA cohort and the reference cohort) in the period 2001-2010, and using the SCHR we studied healthcare utilisation by each individual including hospitalisations and outpatient visits to physicians, nurses and physiotherapists (both in general practice and specialised care). When a subject died or relocated out of the county, data were censored from that time.

\section{Statistical analysis}

We analysed the annual mean number of hospitalisations in total and at a clinic of rheumatology/internal medicine or orthopaedics during the 10-year time frame. We also analysed


Figure 1 Healthcare utilisation in patients with rheumatoid arthritis (RA) and matched referents from the general population, hospitalisation and outpatient visits to physician, nurse and physiotherapist. The y-axes show the mean number of visits per subject per calendar year. 
the mean number of outpatient clinic visits to physicians (including general practitioners), nurses and physiotherapists for each calendar year. To evaluate possible trends relative to the background population, we calculated the ratio of the mean number of visits between the RA cohort and reference cohort for each calendar year and performed a test for trend across ordered groups. We considered a two-tailed $p$ value of 0.05 or less to be significant (Stata software V.11.2).

The study was approved by the regional ethics committee of Lund University.

\section{RESULTS}

General description of cohorts

At the beginning of the study period (1 January 2001), the RA cohort consisted of 3977 subjects (mean age 62.7 years, $72.7 \%$ women). By the end of the study period (31 December 2010), 2471 subjects (62.1\%) were still alive and resident in the Region Skåne. During follow-up, 1417 (35.6\%) subjects had died, 13 of whom had died after they had relocated, and 89 (2.2\%) (still alive) had relocated out of the county (see online appendix A).

Of the 7954 matched referents, 1810 (22.8\%) had died by end of the study period and 237 (3.0\%) (still alive) had relocated from the Region Skåne. Hence, by the end of the study period, 5907 subjects (74.3\%) remained alive and resident in the county (see online appendix B).

\section{Hospitalisations}

The annual mean number of hospitalisations in the RA cohort tended not to increase or decrease during follow-up (figure 1). However, there was a statistically significant trend for a reduced hospitalisation ratio between patients with RA and their reference subjects, both in men $(-27 \%, p=0.01)$ and women $(-28 \%, p=0.004)$ (table 1$)$, suggesting a relative decrease in the number of hospitalisations among patients with RA compared with the general population.

The ratios for hospitalisations at a clinic of rheumatology or internal medicine displayed no trend in men $(-16 \%, p=0.13)$, but declined in women $(-51 \%, p=0.004)$ (table 1, figure 2). The hospitalisation ratio to an orthopaedic clinic decreased in both male $(-39 \%, p=0.006)$ and female $(-36 \%, p=0.02)$ patients (table 1 , figure 2 ).

\section{Outpatient healthcare}

Visits to a physician

During follow-up, the annual mean number of outpatient visits to a physician decreased by approximately one visit in both male and female patients with RA (figure 1). The ratios between patients with RA and reference subjects also decreased for both men $(-29 \%, p=0.005)$ and women $(-16 \%, p=0.003)$ (table 1).

The outpatient consultation ratio to a rheumatologist and/or specialist in internal medicine suggested no significant trends (table 1, figure 2). The consultation ratio to a specialist in orthopaedics tended to decrease in women $(-19 \%, p=0.04)$ (table 1).

\section{Visits to a nurse}

The mean number of visits to nurses (primary care and specialised care) increased for both male and female patients with $\mathrm{RA}$, as well as for reference subjects (figure 1). However, the consultation ratio between both male $(-34 \%, p=0.009)$ and female $(-18 \%, p=0.01)$ patients with $\mathrm{RA}$ and their reference subjects decreased during follow-up (table 1).

\section{Visits to a physiotherapist}

The total number of consultations with physiotherapists (primary care and specialised care) decreased from an annual mean of 5.5 per female patient in 2001 to 3.4 in 2010 (figure 1). The pattern in male patients was similar. The physiotherapy consultation ratios between patients with RA and reference subjects decreased during follow-up in both men $(-34 \%, p=0.01)$ and women $(-28 \%, p=0.004$, table 1$)$.

Table 1 Healthcare utilisation ratio: rheumatoid arthritis (RA) cohort compared with matched referents during the 10-year follow-up

\begin{tabular}{|c|c|c|c|c|c|c|c|c|c|c|c|c|}
\hline & 2001 & 2002 & 2003 & 2004 & 2005 & 2006 & 2007 & 2008 & 2009 & 2010 & Change $(\%)^{*}$ & p Value for trendt \\
\hline \multicolumn{13}{|l|}{ Men } \\
\hline Hospitalisations total & 2.35 & 2.12 & 2.40 & 2.07 & 1.76 & 2.16 & 2.02 & 1.76 & 1.70 & 1.72 & -27 & 0.01 \\
\hline Rheumatology/internal medicine & 3.12 & 2.44 & 3.58 & 2.71 & 1.94 & 2.73 & 2.58 & 2.19 & 1.79 & 2.63 & -16 & 0.13 \\
\hline Orthopaedics & 3.82 & 3.74 & 4.51 & 3.66 & 3.26 & 2.86 & 2.54 & 2.91 & 2.75 & 2.33 & -39 & 0.006 \\
\hline Outpatient physician total & 2.41 & 2.18 & 2.06 & 1.94 & 1.98 & 2.01 & 1.86 & 1.87 & 1.79 & 1.70 & -29 & 0.005 \\
\hline Rheumatology/internal medicine & 4.36 & 5.00 & 4.75 & 4.45 & 4.50 & 5.10 & 4.73 & 4.52 & 4.11 & 3.56 & -18 & 0.25 \\
\hline Orthopaedics & 3.78 & 3.61 & 3.51 & 2.58 & 4.49 & 3.99 & 4.22 & 3.00 & 3.22 & 2.91 & -23 & 0.39 \\
\hline Outpatient nurse total & 2.63 & 2.30 & 2.12 & 2.17 & 2.32 & 1.77 & 1.75 & 1.64 & 1.66 & 1.73 & -34 & 0.009 \\
\hline Outpatient physiotherapist total & 2.92 & 3.28 & 2.92 & 2.47 & 2.24 & 2.46 & 2.67 & 2.24 & 2.23 & 1.94 & -34 & 0.01 \\
\hline \multicolumn{13}{|l|}{ Women } \\
\hline Hospitalisations total & 2.79 & 2.58 & 2.54 & 2.62 & 2.30 & 2.23 & 2.23 & 2.13 & 2.20 & 2.02 & -28 & 0.004 \\
\hline Rheumatology/internal medicine & 4.77 & 3.60 & 3.50 & 3.62 & 2.94 & 2.99 & 2.80 & 2.74 & 2.50 & 2.36 & -51 & 0.004 \\
\hline Orthopaedics & 3.89 & 3.96 & 2.90 & 2.96 & 3.10 & 2.24 & 2.74 & 2.18 & 2.47 & 2.48 & -36 & 0.02 \\
\hline Outpatient physician total & 2.11 & 2.09 & 1.96 & 1.96 & 1.95 & 1.91 & 1.88 & 1.82 & 1.82 & 1.77 & -16 & 0.003 \\
\hline Rheumatology/internal medicine & 3.65 & 4.40 & 4.64 & 4.80 & 4.34 & 5.31 & 4.68 & 4.44 & 5.10 & 4.88 & +34 & 0.055 \\
\hline Orthopaedics & 3.65 & 3.63 & 3.88 & 3.96 & 3.85 & 3.78 & 3.57 & 3.28 & 3.11 & 2.96 & -19 & 0.04 \\
\hline Outpatient nurse total & 2.40 & 2.44 & 2.28 & 2.14 & 2.08 & 2.10 & 2.14 & 1.83 & 2.10 & 1.98 & -18 & 0.01 \\
\hline Outpatient physiotherapist total & 2.44 & 2.38 & 2.48 & 2.34 & 2.19 & 2.09 & 2.10 & 2.02 & 1.85 & 1.76 & -28 & 0.004 \\
\hline
\end{tabular}

Values are the mean number of visits per calendar year per RA patient divided by the corresponding mean for reference subjects. A ratio of $>1$ indicates more healthcare utilisation in patients with RA.

${ }^{*}$ Change in the ratio as a percentage from 2001 to 2010.

tp Value from test for trend, ordered by rank. 

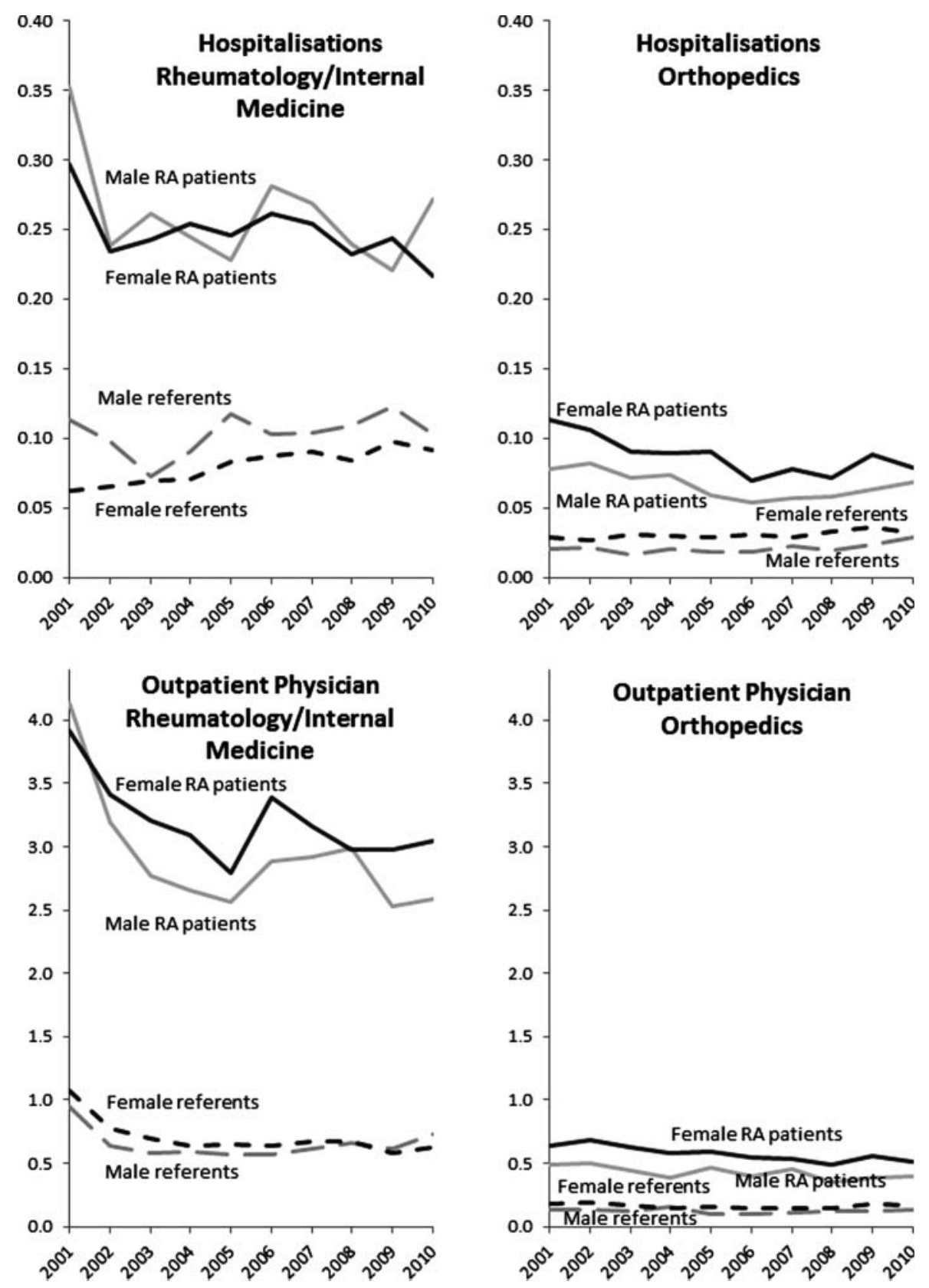

Figure 2 Healthcare utilisation in patients with rheumatoid arthritis (RA) and matched referents from the general population, specialised hospitalisation and outpatient visits to physician. The $y$-axes show the mean number of visits per subject per calendar year.

\section{DISCUSSION}

Major changes in the treatment of RA have occurred during the first decade of the 21st century. Using Swedish observational data, we found both inpatient and outpatient healthcare utilisation by a cohort of patients with RA to have decreased relative to the general population.

Increased need for healthcare is to be expected in an ageing group of people whether they have a chronic disease or not. ${ }^{16}$ We observed such tendencies in our reference subjects, but not to the same degree in the RA cohort. The decrease in the gap in healthcare utilisation between the RA cohort and referents seems largely to be explained by fewer visits by patients with RA to physicians outside the field of rheumatology, where we also saw fewer visits to physiotherapists and nurses. Less utilisation of physician care in the USA has previously been reported, ${ }^{17}$ and patients monitored closely in anti-TNF studies have also been reported to have less frequent visits to physicians. ${ }^{13}$ However, the explanation for these findings was traced to administration of anti-TNF therapy, and visits to physicians increased slightly for patients receiving therapy subcutaneously. $^{13} 14$ In our study setting, monitoring of eventual biological treatment is integrated into the specialised outpatient care by both rheumatologists and nurses. Previous studies on secular trends on utilisation of nurses and physiotherapists are lacking, although this kind of information is important to understand and monitor the burden of RA.

Earlier studies on healthcare utilisation in RA have mainly been based on investigation of cost of disease, analysis of the cost of pharmacological treatment, or burden of disease. Importantly, observational studies based on existing data from the healthcare system have been encouraged to add value and new perspectives. ${ }^{14} 18$ Our results are based on actual observed 
healthcare utilisation and placed in context by comparison with reference subjects from the general population. However, there are also important limitations. In our cohort, we followed $\sim 80 \%$ of the total expected number of patients with RA resident in the Region Skåne. ${ }^{5}$ The remaining $20 \%$ of patients with RA, not captured, were predominantly patients who were regularly seen by private practising rheumatologists or did not consult a rheumatologist during 1998-2001, and their disease activity was probably less severe. ${ }^{19} 20$ Further, there is a healthy survivor effect-that is, patients with RA who survive the study period are, in general, healthier than those who do not. However, we can expect the same phenomenon in the reference cohort. Ultimately, we would have preferred to study a dynamic RA cohort compared with a dynamic reference cohort. However, increased reporting of diagnostic codes to the register during the study period, primarily from primary care, precluded such a study design, as it would introduce selection bias (probably capturing more and less severe RA cases, which may have affected the results). Finally, it is plausible that changes in the healthcare system (eg, more ambulatory treatment of certain conditions) during this decade may have affected the healthcare utilisation by patients with chronic and non-chronic diseases differently.

To conclude, during the 21st century (representing modern rheumatology), we found reduced overall healthcare utilisation in a cohort of patients with RA compared with the general population.

Acknowledgements We would like to acknowledge statistical support from Ljuba Kedza, RC Syd, Sweden.

Contributors All authors participated in planning and performing this study. All authors confirm that they have read and approved the content presented in the final manuscript for submission.

Funding This work was supported by the Norrbacka-Eugenia Foundation, the Capio's Research Foundation, the Swedish Research Council, the Region Skåne and the Faculty of Medicine, Lund University, Sweden.

Competing interests None.

Ethics approval The regional ethics committee of Lund University.

Provenance and peer review Not commissioned; externally peer reviewed.

\section{REFERENCES}

1. van Vollenhoven RF, Geborek P, Forslind $\mathrm{K}$, et al. Conventional combination treatment versus biological treatment in methotrexate-refractory early rheumatoid arthritis: 2 year follow-up of the randomised, non-blinded, parallel-group Swefot trial. Lancet 2012;379:1712-20.
2. Smolen JS, Aletaha D, Bïlsma JW, et al. Treating rheumatoid arthritis to target: recommendations of an international task force. Ann Rheum Dis 2010;69:631-7.

3. Smolen JS, Landewe R, Breedveld FC, et al. EULAR recommendations for the management of rheumatoid arthritis with synthetic and biological disease-modifying antirheumatic drugs. Ann Rheum Dis 2010;69:964-75.

4. Neovius M, Simard JF, Askling J. Nationwide prevalence of rheumatoid arthritis and penetration of disease-modifying drugs in Sweden. Ann Rheum Dis 2011;70:624-9.

5. Englund $\mathbf{M}$, Joud A, Geborek P, et al. Prevalence and incidence of rheumatoid arthritis in southern Sweden 2008 and their relation to prescribed biologics. Rheumatology (Oxford) 2010;49:1563-9.

6. Olofsson T, Englund M, Saxne T, et al. Decrease in sick leave among patients with rheumatoid arthritis in the first 12 months after start of treatment with tumour necrosis factor antagonists: a population-based controlled cohort study. Ann Rheum Dis 2010;69:2131-6.

7. ter Wee MM, Lems WF, Usan $\mathrm{H}$, et al. The effect of biological agents on work participation in rheumatoid arthritis patients: a systematic review. Ann Rheum Dis 2012;71:161-71.

8. Bansback N, Marra CA, Finckh A, et al. The economics of treatment in early rheumatoid arthritis. Best Pract Res Clin Rheumatol 2009;23:83-92.

9. Bansback NJ, Regier DA, Ara R, et al. An overview of economic evaluations for drugs used in rheumatoid arthritis: focus on tumour necrosis factor-alpha antagonists. Drugs 2005;65:473-96.

10. Haraoui B, Pope J. Treatment of early rheumatoid arthritis: concepts in management. Semin Arthritis Rheum 2011;40:371-88.

11. Hekmat K, Jacobsson L, Nilsson JA, et al. Decrease in the incidence of total hip arthroplasties in patients with rheumatoid arthritis - results from a well defined population in south Sweden. Arthritis Res Ther 2011;13:R67.

12. Shourt CA, Crowson CS, Gabriel SE, et al. Orthopedic surgery among patients with rheumatoid arthritis 1980-2007: a population-based study focused on surgery rates, sex, and mortality. J Rheumatol 2012;39:481-5.

13. Sandhu RS, Treharne GJ, Douglas KM, et al. The impact of anti-tumour necrosis factor therapy for rheumatoid arthritis on the use of other drugs and hospital resources in a pragmatic setting. Musculoskeletal Care 2006;4:204-22.

14. Fautrel B, Verstappen SM, Boonen A. Economic consequences and potential benefits. Best Pract Res Clin Rheumatol 2011;25:607-24.

15. Strombeck B, Jacobsson LT, Bremander A, et al. Patients with ankylosing spondylitis have increased sick leave-a registry-based case-control study over 7 yrs. Rheumatology (Oxford) 2009;48:289-92.


utilization among older people $(60+)$ : focusing on ADL dependency and risk of depression. Arch Gerontol Geriatr 2012;54:e349-63.

17. Joyce GF, Goldman DP, Karaca-Mandic P, et al. Impact of specialty drugs on the use of other medical services. Am J Manag Care 2008;14:821-8.

18. Kobelt G. Economic studies in rheumatology: data, perspectives, challenges. Rheumatology (Oxford) 2012;51:208-9.

19. Bergstrom U, Jacobsson LT, Turesson C. Cardiovascular morbidity and mortality remain similar in two cohorts of patients with long-standing rheumatoid arthritis seen in 1978 and 1995 in Malmo, Sweden. Rheumatology (Oxford) 2009;48:1600-5.

20. Book C, Saxne T, Jacobsson LT. Prediction of mortality in rheumatoid arthritis based on disease activity markers. J Rheumatol 2005;32:430-4. 